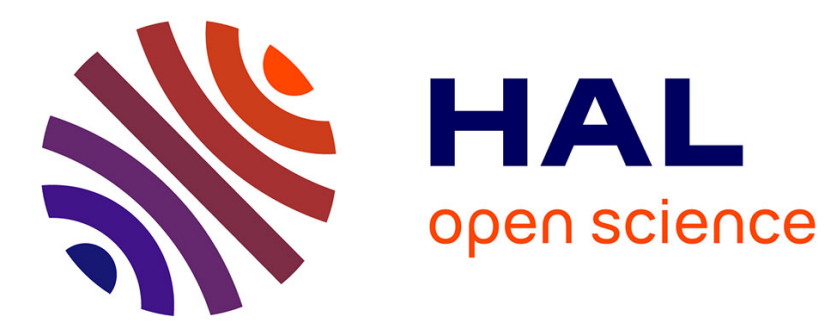

\title{
"La mère de Berlin ", représentation de Louise Schroeder dans la presse écrite (1947-1957)
}

\author{
Agathe Bernier-Monod
}

\section{To cite this version:}

Agathe Bernier-Monod. "La mère de Berlin ", représentation de Louise Schroeder dans la presse écrite (1947-1957). Allemagne d'aujourd'hui: revue francaise d'information sur l'Allemagne, 2014. hal-02089947

\section{HAL Id: hal-02089947}

\section{https://hal-normandie-univ.archives-ouvertes.fr/hal-02089947}

Submitted on 4 Apr 2019

HAL is a multi-disciplinary open access archive for the deposit and dissemination of scientific research documents, whether they are published or not. The documents may come from teaching and research institutions in France or abroad, or from public or private research centers.
L'archive ouverte pluridisciplinaire HAL, est destinée au dépôt et à la diffusion de documents scientifiques de niveau recherche, publiés ou non, émanant des établissements d'enseignement et de recherche français ou étrangers, des laboratoires publics ou privés. 


\section{Agathe Bernier-Monod : " La mère de Berlin », représentation de Louise Schroeder dans la presse écrite (1947-1957).}

\section{Briatte-Peters, Anne-Laure/Danckaerts, François (dir) : Les femmes politiques dans la vie politique allemande depuis 1945, Allemagne d'aujourd'hui, janvier-mars 2014, p. 74-82.}

Seule femme maire de Berlin à ce jour et une des rares femmes à accéder à de hautes responsabilités dans l'Allemagne de l'après-guerre, Louise Schroeder est aujourd'hui largement oubliée de l'historiographie comme du grand public. Lorsqu'elle accède au poste de maire intérimaire en mai 1947, cette sociale-démocrate âgée de 60 ans a déjà une longue carrière politique derrière elle. Elle demeure à la tête de la ville jusqu'en décembre 1948, avant d'exercer la fonction d'adjointe au maire de décembre 1948 à janvier 1951, et de représenter Berlin au Bundestag de 1949 à 1957. Le cas Schroeder est hors du commun du fait de son sexe, de son statut intermédiaire d'intérimaire et du contexte tumultueux du Berlin d'avant la division.

Cette analyse tentera de répondre aux deux questions suivantes : quelle image de Louise Schroeder la presse de l'après-guerre a-t-elle véhiculée ? Dans quelle mesure les stéréotypes genrés dominaient-ils son image médiatique ?

Le corpus de presse étudié est pour Berlin-Ouest le Tagesspiegel, pour la zone d'occupation soviétique Neues Deutschland, ainsi que le Berliner Zeitung, qui détaille davantage la politique municipale. Enfin, le quotidien Frankfurter Rundschau et les hebdomadaires Der Spiegel et Die Zeit donnent un aperçu de la perspective jetée à l'Ouest sur Louise Schroeder, de mai 1947, date de la prise de ses fonctions comme maire de Berlin, à sa mort en juin 1957. Plus de cent articles abordant son action ou esquissant son portrait ont été examinés.

Ce travail s'appuie sur des catégories élaborées par Gertrude Robinson et Armande SaintJean dans leur analyse de la couverture médiatique des femmes politiques canadiennes suivant un prisme générationnel. Les deux chercheuses ont observé que les reportages portant sur les «premières femmes » politiques, nées avant 1930, adoptaient une «approche biologique » soulignant leur apparence physique et leurs relations familiales ${ }^{1}$. Cette valorisation de

\footnotetext{
${ }^{1}$ Robinson, Gertrude J., Saint-Jean, Armande : «Women Politicians and their Media Coverage. A Generational Analysis » dans Megyery, Kathy (dir) : Women in Canadian Politics. Towards Equity in Representation, Toronto/Oxford : Dundurn Press, 1991, pp. 127-169, ici p. 135.
} 
«1'histoire-cadre » au détriment de leur engagement politique s'applique bien au cas Schroeder. Toutefois, en se voyant décerner le titre de «mère de Berlin » par les médias, Louise Schroeder revêtait une dimension symbolique transcendant le schéma ordinairement associé à la « première femme ». Enfin, la presse de l'Est, s'efforçant d'endiguer ce mythe, construisit un contre-récit présentant Louise Schroeder comme une «mauvaise mère ».

\section{Le charme discret de Louise Schroeder}

Dans la presse, Louise Schroeder apparaissait comme en net retrait par rapport à Ernst Reuter ou à d'autres hommes politiques sociaux-démocrates de sa stature. Née en 1887 à Altona (Hambourg) dans une famille ouvrière et socialiste, cette sténotypiste de formation adhéra au parti social-démocrate en 1910. Les soirées féminines de la section locale d'Altona-Ottensen lui fournirent l'occasion de développer ses talents oratoires. Élue au bureau de cette section entre 1915 et $1918^{2}$, elle devint ensuite la plus jeune parlementaire de l'Assemblée nationale de Weimar, puis siégea sans interruption au Reichstag de 1920 à 1933. Comptant parmi les femmes entendues au Parlement, elle s'investit particulièrement dans le domaine des affaires sociales et du droit du travail. La loi de protection des mères (Mutterschutzgesetz) de 1927 a ainsi été communément appelée «lex Schroeder». Elle fonda également l'association caritative Arbeiterwohlfahrt, avec Marie Juchacz en 1919. Suspectée de haute trahison pendant la période nazie, elle se retrouva sans ressource et continua de fréquenter les cercles sociaux-démocrates clandestins de Hambourg, puis de Berlin. Au lendemain de la guerre, elle devint vice-présidente du SPD du Land de Berlin et fit partie du bureau fédéral du parti de 1946 à $1957^{3}$. Adjointe d'Ernst Reuter et plus consensuelle que ce dernier, elle apparut comme sa remplaçante idéale lors de l'invalidation de son élection par les Soviétiques.

Elle resta toutefois dans l'ombre du maire officiellement élu. Une recherche en ligne sur les sites du Spiegel et de la Zeit révèle ainsi que ces deux journaux consacrèrent, entre 1947 et 1957, période retenue pour cette étude, deux fois plus d'articles à Ernst Reuter qu'à Louise

\footnotetext{
2 Marquardt, Regine : Das Ja zur Politik: Frauen im Deutschen Bundestag (1949 - 1961); ausgewählte Biographien. Opladen : Leske + Budrich, 1999, p. 108.

3 «Louise Schroeder» dans Allgemeine deutsche Biographie \& Neue deutsche Biographie (Digitale Register), pp. 569-571.

http://daten.digitalesammlungen.de/ db/0001/bsb00019558/images/index.html?id=00019558\&fip=yztseayafsdrsdasxssdaseayayztsxs\& $\underline{\text { no }=1 \& \text { seite }=589}$.
} 
Schroeder ${ }^{4}$. Cette disproportion suggère que Schroeder a fait l'objet de la même sousreprésentation médiatique que la plupart des politiciennes de sa génération. Dans un article décrivant l'évolution de couverture des femmes politiques par la presse allemande de 1945 à nos jours, Birgit Meyer observe en effet que les années 1950 ont largement passé sous silence les figures féminines, même de premier plan. ${ }^{5}$

Ce silence relatif tient probablement aussi aux circonstances troublées de l'exercice de ses fonctions. Il faut préciser que la municipalité de Berlin, placée sous les ordres des quatre puissances occupantes, disposait alors d'une marge de manœuvre extrêmement réduite. Louise Schroeder occupait en outre un poste de remplacement : de mai 1947 à décembre 1948 - et donc pendant les six premiers mois du blocus -, elle remplaça Otto Ostrowski, déchu pour avoir refusé de licencier des fonctionnaires membres du SED (parti unifié né en 1946 de la fusion forcée entre les partis communiste et social-démocrate), puis Ernst Reuter. Si les responsabilités revenaient officiellement à Schroeder, Reuter dirigeait en coulisse et demeurait l'interlocuteur privilégié des Alliés américains. ${ }^{6}$ Le début du blocus de Berlin correspondit enfin pour elle à une période de maladie entraînant son absence prolongée. Il se peut donc que la position délicate de la maire ait incité un service de presse efficace et des journalistes bienveillants à ne pas trop la placer sous les feux de la rampe afin de ne pas susciter de mise en cause de sa légitimité. Schroeder suivait elle-même de près sa couverture médiatique, tout particulièrement les attaques que la presse de la zone d'occupation soviétique portait à la municipalité ${ }^{7}$. On peut supposer que cette grande discrétion desservait l'image de la maire et ne rendait pas pleinement justice à son action. En effet, comme l'affirme Joachim Raschke, théoricien des mouvements sociaux : «La pire nouvelle est l'absence de nouvelles. » ${ }^{8}$

4

http://www.spiegel.de/suche/index.html?suchbegriff=\%22louise+schroeder\%22\&quellenGroup=SP \&fromDate=01. $01.1947 \&$ toDate $=31.12 .1957$

http://www.spiegel.de/suche/index.html?suchbegriff=\%22ernst+reuter\%22\&quellenGroup=SP\&fromDate=01.01.19 47\&toDate $=31.12 .1957$

http://www.zeit.de/suche/index?q=\%22louise+schroeder\%22\&from=01.01.1947\&to=31.12.1957 ;

http://www.zeit.de/suche/index?q=\%22ernst+reuter\%22\&from=01.01.1947\&to=31.12.1957 (26.09.2013)

${ }_{5}^{5}$ Meyer, Birgit : «"Nachts, wenn der Generalsekretär weint" - Politikerinnen in der Presse » dans Frauen in Politik und Medien. Aus Politik und Zeitgeschichte 50 (7 décembre 2009), pp. 9-15.

${ }^{6}$ Koerfer, Marthina : Louise Schroeder: eine Frau in den Wirren deutscher Politik. Berlin : Presse- und Informationsamt des Landes Berlin, 1987, p. 43.

${ }^{7}$ Landesarchiv Berlin, C Rep 101-139 Oberbürgermeister. Verbindung mit Presse und Rundfunk. Magistrat von Groß-Berlin, Presseamt. Lettre à Louise Schroeder, octobre 1947.

8 «Die schlechteste Nachricht ist keine Nachricht.» Raschke, Joachim : Soziale Bewegungen: ein historischsystematischer Grundriß. Campus : Francfort, New York 1985, p. 344. 
Le plus souvent élogieux, les papiers qui lui étaient consacrés avaient largement recours à des stéréotypes genrés. La mention de son apparence physique était ainsi récurrente. La Frankfurter Rundschau, qui ne la nommait généralement qu'en marge de ses articles, publiait volontiers des photographies d'elle lors de sa participation aux réunions de ministres-présidents de Länder. ${ }^{9}$ Les journalistes ne manquaient pas d'évoquer au passage « la robe noire toute simple » ou encore «la bonté personnifiée douée de charme aux cheveux gris ${ }^{10}$, démontrant ainsi que Louise Schroeder défiait l'idée reçue de la masculinité inhérente aux femmes politiques. Le Spiegel revenait quant à lui sur son apparence modeste, laissant même entendre que la représentante de Berlin manquait d'élégance. ${ }^{11}$ Son mode de vie semble aussi avoir éveillé un intérêt certain: malgré sa fonction prestigieuse, elle continua à sous-louer une humble chambre dans le quartier de Tempelhof. ${ }^{12}$ Ces ébauches de portraits mêlaient généralement caractérisation physique et caractérisation morale laudative. Un leitmotiv de ces descriptions réside dans le contraste que formaient son corps frêle et sa force de caractère, qui trouve son expression dans l'emploi de l'allitération en z: «zart» («délicat»), «zierlich» («menue »), «zerbrechlich» $\left(\right.$ fragile ») et «zäh» («coriace »). ${ }^{13}$ D’après certains rédacteurs, c'est justement cet équilibre rare entre fragilité, force et simplicité qui faisait d'elle une habile négociatrice, à même de contenir les tensions entre Alliés occidentaux et soviétiques ${ }^{14}$.

\section{Une maternité symbolique}

Un attribut féminin indissociable de la représentation de Louise Schroeder se révèle être la maternité. Le titre de «mère de Berlin » («die Mutter Berlins ») ${ }^{15}$ de même que ses vertus

\footnotetext{
9 «Treffen der Ministerpräsidenten ». Frankfurter Rundschau, 7 juin 1948 ; «Einstimmigkeit in Koblenz. Deutsche Gegenvorschläge - Vorbehalte der Ministerpräsidenten. », ibid., 10 juillet 1948.

10 «einem schlichten schwarzen Kleid», «Drei Bürgermeister berichteten». Der Tagesspiegel, 12 juin 1947 ; «die personifizierte Gütigkeit mit grauhaarigem Charme » "Vorläufig eins zu eins ». Der Spiegel, 26 juin 1948. http://www.spiegel.de/spiegel/print/d-44417683.html (09.01.2013).

${ }_{11}$ « Späte Liebe ». Der Spiegel, 17 mai 1947 ; «Wie 45. Höchstens 14 friedliche Tage ». Der Spiegel, 3 juillet 1948.

12 «Zu Louise Schroeders Ehrentag ». Der Tagesspiegel, 2 avril 1957.

${ }^{13}$ «Vorläufig eins zu eins », op. cit. ; "Köpfe deutscher Bürgermeister ». Die Zeit, 15 juillet 1948, http://www.zeit.de/1948/29/koepfe-deutscher-buergermeister (01.08.2013)

14 «Die Frauen zerbrechen die Blockade ». Die Zeit, 26 août 1948. http://www.zeit.de/1948/35/frauen-zerbrechendie-blockade/seite-2 (01.08.2013).

$\frac{15}{15}$ «estdeutschlands Aufgabe ». Der Tagesspiegel, 27 juillet $1948 ;$ «Die Hintergründe der Koblenzer Beschlüsse der westdeutschen Ministerpräsidenten ». Der Tagesspiegel, 3 août 1948 ; «Doppelt oder nichts? Brauchen wir einen neuen Kulturbund? ». Neues Deutschland, 28 juillet 1948.
} 
maternelles supposées ${ }^{16}$ apparaissent dans de nombreux articles. ${ }^{17}$ Les qualités morales qui lui étaient attribuées - bonté, douceur, amour, dévouement confinant à l'abnégation ${ }^{18}-$ sont elles aussi couramment associées à la maternité. «Elle est dans sa relation aux Berlinois, comme une mère qui se fait du souci pour son enfant malade ${ }^{19}$, peut-on lire dans la Zeit. À en croire Gertrude Robinson et Armande Saint-Jean, la mention de la maternité d'une femme politique et l'assimilation de ses compétences à des talents maternels n'ont rien d'exceptionnel, dans la mesure où sa vie privée et ses relations familiales dominent généralement son image. ${ }^{20}$ Birgit Meyer constate également que les médias des années 1950 et 1960 recouraient largement au cliché de la «bonne mère » pour dépeindre les femmes politiques. ${ }^{21}$ Louise Schroeder était toutefois célibataire et sans enfant ; la notion de maternité, omniprésente dans sa représentation médiatique, assumait donc une fonction toute symbolique. Le titre de «mère de Berlin » érigeait ainsi Louise Schroeder en représentante de toutes les mères berlinoises ayant œuvré à la sauvegarde de la ville, les « femmes des ruines » (Trümmerfrauen) notamment. ${ }^{22}$

Plusieurs explications peuvent être apportées à la constitution de cette image. Dans ses interventions au conseil municipal et au Bundestag, Louise Schroeder se posait elle-même en porte-parole de la détresse berlinoise et en médiatrice entre les habitants et les autorités occupantes des zones occidentales. ${ }^{23}$ Cette caractérisation faisait aussi référence à la grande popularité dont elle bénéficiait auprès des Berlinois. Les très nombreuses lettres de remerciements ou demandes d'aide qui lui étaient adressées ${ }^{24}$ témoignent de la confiance qui lui était accordée. Cette relation de proximité a pu être suscitée par les origines modestes de la maire, fille d'un ouvrier en bâtiment d'Altona, par la misère dans laquelle elle avait vécu pendant

\footnotetext{
16 «Köpfe deutscher Bürgermeister », op. cit.

17 Sur l'association à la maternité des femmes engagées dans la politique municipale cf. Wolff, Kerstin : Stadtmütter. Bürgerliche Frauen und ihr Einfluss auf die Kommunalpolitik im 19. Jahrhundert (1860-1900), Königstein/Taunus : Helmer, 2003.

18 «Vorläufig eins zu eins », op. cit.; «Köpfe deutscher Bürgermeister». op. cit..

19 «Sie ist in ihrem Verhältnis zu den Berlinern wie eine Mutter, die sich um ihr krankes Kind sorgt. », «Die Frauen zerbrechen die Blockade », op. cit..

${ }^{20}$ Robinson, Gertrude J., Saint-Jean, Armande, op. cit., p. 135.

21 Meyer, Birgit, op. cit., p. 10.

22 «Die Frauen zerbrechen die Blockade », op.cit..

${ }^{23}$ Cf. Schroeder, Louise : Ein Jahr Stellvertretender Oberbürgermeister von Groß-Berlin. Reden und Ansprachen in den Stadtverordnetenversammlungen von Groß-Berlin vom 8. Mai 1947 bis 8. Mai 1948. Berlin, 1949 , 12 juin $1947,33^{\text {ème }}$ séance, p. 5 ; Verhandlungen des Deutschen Bundestages, 30 septembre 1949, $11^{\text {ème }}$ séance, pp. 229 C$231 \mathrm{~A}$.

${ }^{24}$ Cf. par exemple : Landesarchiv Berlin, C Rep 101-135, «Bittzuschriften und Begrüßungen zur Amerika-Reise von Bürgermeisterin Louise Schroeder ».
} 
la guerre et par son apparence de grande simplicité. C'est elle que le SPD choisit pour adresser une lettre de campagne à l'ensemble des Berlinois lors de l'élection de décembre 1948, alors qu'elle se trouvait à l'hôpital. ${ }^{25}$

Cette exaltation de la maternité s'explique aussi par le contexte culturel du tournant des années 1940-1950, marqué par le retour de la famille comme lieu de projection des aspirations individuelles. ${ }^{26}$ A la faveur de cette évolution, les partis politiques allemands ne s'adressaient aux électrices qu'en leur qualité de mères potentielles. ${ }^{27}$ Le cas Schroeder semble donc indiquer que les politiciennes aussi se devaient d'entrer dans cette catégorie

La maternité était également une notion omniprésente dans les associations de femmes constituées en 1945 et permettait aux dirigeantes, le plus souvent plus âgées et instruites, d'asseoir leur autorité face aux plus jeunes. ${ }^{28}$ En ce sens, on peut identifier une parenté entre les associations de femmes de l'après-guerre et le premier féminisme allemand. ${ }^{29}$ Les «Frauenverbände» semblent en effet avoir repris à leur compte un élément argumentatif du mouvement des femmes de la République de Weimar, qui présentait l'engagement politique féminin comme une forme de «maternité organisée ${ }^{30}$. La participation politique des femmes devait, au sens de ses partisanes, permettre d'étendre les vertus maternelles à la société entière. ${ }^{31}$ La perception des femmes politiques comme «mères » peut donc éventuellement découler de ces représentations.

Enfin, force est de constater surtout la dimension apolitique de cette caractérisation. En effet, les rares critiques formulées à l'encontre de Schroeder mettaient en doute ses compétences politiques. Elles la décrivaient comme une gestionnaire, largement épaulée par Reuter, capable certes d'assumer des fonctions représentatives et de régler les affaires courantes, mais manquant

25 «Noch von ihrem Krankenlager schrieb Frau Louise Schroeder, Berlins amtierende Oberbürgermeisterin, an uns zu diesen Wahlen. » Der Tagesspiegel, 5 décembre 1948.

${ }^{26}$ Schelsky, Helmut: Wandlungen der deutschen Familie in der Gegenwart: Darstellung und Deutung einer empirisch-soziologischen Tatbestandsaufnahme, Stuttgart : Enke, 1955, p. 91.

${ }^{27}$ Perrot, Michelle : «Sur le front des sexes: un combat douteux ». Vingtième Siècle. Revue d'histoire 3 , n ${ }^{\circ} 1$ (1984), p. 73.

${ }^{28}$ Möding, Nori : «Die Stunde der Frauen », dans Broszat, Martin, Henke, Klaus-Dietmar, Woller, Hans (dir.): Von Stalingrad zur Währungsreform. Zur Sozialgeschichte des Umbruchs in Deutschland, Munich : Oldenbourg, 1988, pp. 619-648, ici p. 640 .

${ }^{29}$ Sur cette relation de filiation, voir la contribution de Kerstin Wolff dans ce volume.

30 Stoehr, Irene : «"Organisierte Mütterlichkeit". Zur Politik der deutschen Frauenbewegung um 1900 », dans Hausen, Karin (dir): Frauen suchen ihre Geschichte. Historische Studien zum 19. Und 20. Jahrhundert, Munich : Beck, 1983, pp. 221-249.

31 Gerhard, Ute, Klausmann, Christina, Wischermann, Ulla, « Neue Staatsbürgerinnen - die deutsche Frauenbewegung in der Weimarer Republik», dans Gerhard, Ute (dir.) : Feminismus und Demokratie, Europäische Frauenbewegungen der 1920er Jahre, Königstein/Taunus : Helmer, 2001, p. 186. 
de l'envergure nécessaire à la prise de décisions. Le Spiegel écrivait ainsi qu'elle n'était «pas une pointure politique $» .^{32}$ Ces attaques portaient principalement sur ses erreurs stratégiques supposées quant au statut revenant à Berlin-Ouest dans la République en devenir. Son intervention lors de la conférence du Rittersturz de juillet 1948, qui devait préparer la création d'un État ouest-allemand, fut jugée funeste. ${ }^{33}$ Espérant encore parvenir à un accord en vue de l'unification du pays, Schroeder trouva prématuré d'envisager l'inclusion de Berlin-Ouest dans la République fédérale. Ernst Reuter revint sur cette position huit jours plus tard, dans le cadre de la conférence de Niederwald.

La politique menée par Louise Schroeder à la tête de Berlin visait principalement à atténuer la situation de pénurie généralisée de la ville. Il s'agissait d'une politique sociale axée sur des besoins tout à fait matériels, qui semblaient éloignés des intérêts de la «haute politique » (finances, défense, affaires étrangères), demeurée une chasse gardée masculine. ${ }^{35}$ La dimension politique de l'action de Louise Schroeder pouvait donc être aisément contestée. L'analyse qu'Annette Kuhn livre de la contribution des femmes à la reconstruction s'applique bien à la perception médiatique de la maire :

[...] entre 1945 et 1948, la séparation entre politique et quotidien [semblait] abolie. [...] La politique dans ces premières années était une politique de la pomme de terre, et la politique de la pomme de terre était l'affaire des femmes. ${ }^{36}$

Louise Schroeder ne fut donc pas pleinement prise au sérieux sur le plan politique, en dépit des efforts qu'elle déploya pour faire figure d'experte dans le domaine des affaires sociales et de la santé publique ${ }^{37}$. Déconsidérés car associés à des métiers perçus comme féminins, ces secteurs n'étaient pas reconnus comme partie prenante de la «haute politique». Une telle position revenait à méconnaître le travail effectué par la maire en vue d'assurer la subsistance des

\footnotetext{
32 «Wie 45. Höchstens 14 friedliche Tage ». Der Spiegel, 3 juillet 1948.

33 «Westdeutschlands Aufgabe ». op. cit. ; «Die Hintergründe der Koblenzer Beschlüsse der westdeutschen Ministerpräsidenten ». Der Tagesspiegel, 3 août 1948.

${ }^{35}$ Scott, Joan : « Genre : Une catégorie utile d'analyse historique ». Les Cahiers du GRIF 37, n 1 (1988) : pp. 125153, ici p. 130.

http://www.persee.fr/web/revues/home/prescript/article/grif_0770-6081_1988_num_37_1_1759 (05/10/2013)

${ }^{36}$ « [...] zwischen 1945 und 1948, in der die Trennung von Politik und Alltag aufgehoben schien [...] Politik in diesen frühen Jahren war Kartoffelpolitik, und Kartoffelpolitik war Frauensache. » Annette Kuhn (dir) : Politeia: Szenarien aus der deutschen Geschichte nach 1945 aus Frauensicht. Ausstellungskatalog. Bonn : FrauenMuseum, 1999, p. 75.

37 Landesarchiv Berlin, C Rep 101-174. Ministerpräsidentenkonferenz der deutschen Länder 4-8 juin 1947 ; «Schlussbericht aus München ». Frankfurter Rundschau, 10 juin 1947.
} 
Berlinois de l'Ouest, en termes de mobilisation de ses réseaux et de campagnes de lobbying auprès des Alliés et des maires de grandes villes dans les zones occidentales.

Au lendemain de la guerre, les médias étaient confrontés à une situation nouvelle : pour la première fois, certaines femmes accédaient à d'importantes fonctions en devenant ministres à l'échelle du Land ou maires de grandes villes. ${ }^{38}$ Plutôt que de prendre acte de cette entrée dans la sphère publique et politique et d'envisager les transformations qui en résultaient, la presse présenta leur engagement comme une simple extension de leurs activités dans la sphère privée. C'est sans doute pour cette raison que Louise Schroeder, quoique politicienne professionnelle et aguerrie, demeurait perçue principalement en termes affectifs et émotionnels.

\section{À l'Est : l'élaboration d'un récit alternatif}

Bien moins méliorative, la presse de l'Est entreprit de contrecarrer la popularité de la maire auprès des Berlinois. Elle reprit l'idée de maternité pour la subvertir et affubla Louise Schroeder du sobriquet familier de «Mutter Schroeder $» .{ }^{39}$ L'image de cette dernière subit une dégradation progressive. Avant la réforme monétaire, alors que Berlin était encore susceptible de passer sous l'emprise soviétique, les quotidiens Neues Deutschland et Berliner Zeitung rendaient compte de ses activités dans des articles factuels ou notaient les efforts de conciliation de la maire, soucieuse de rassembler par-delà les zones d'occupation. L'article « Louise Schroeder contre le plan Hoover » rapporte qu'elle se serait défendue de conclure une paix séparée avec l'Ouest : «Seule une paix avec l'ensemble de l'Allemagne est acceptable pour la social-démocratie », aurait-elle déclaré. ${ }^{40}$ L'exacerbation des dissensions régnant au sein de la municipalité et du $\mathrm{SPD}^{41}$ à laquelle procédaient ces deux journaux donnait généralement le beau rôle à Schroeder, qui faisait figure de représentante de la vieille garde marxiste, divergeant du «cours purement

\footnotetext{
${ }^{38}$ Schüller, Elke : Wer stimmt, bestimmt, Elisabeth Selbert und die Frauenpolitik der Nachkriegszeit, Wiesbaden : Hessisches Ministerium für Frauen, Arbeit und Sozialordnung, 1996, p. 23.

39 «Mutter Schroeder reist schon wieder». Berliner Zeitung, 28 juillet 1948.

40 «Nur ein Friede mit Gesamtdeutschland sei für die Sozialdemokratie annehmbar.» «Louise Schroeder gegen den Hoover-Plan ». Berliner Zeitung, 15 juin 1948.

${ }^{41}$ «Neuer Länderchef-Treffen ». Berliner Zeitung, 14 novembre 1947.
} 
anticommuniste du parti $\gg .{ }^{42}$ Plusieurs articles mettaient en avant ses déclarations en faveur du socialisme et de l'unité allemande. ${ }^{43}$

L’introduction de la réforme monétaire dans les zones occidentales de Berlin en juin 1948 marqua un tournant dans la réception de Louise Schroeder à l'Est. À partir de cet événement, Neues Deutschland et le Berliner Zeitung entreprirent un démontage systématique de ses discours. Chacune de ses allocutions faisait l'objet d'une dissection critique. Les mérites qu'elle attribuait à la municipalité du Grand-Berlin ou aux Alliés occidentaux étaient invariablement mis sur le compte des Soviétiques. ${ }^{44}$ Cette stratégie eut pour résultat paradoxal de donner à la maire davantage de visibilité à l'Est qu'à l'Ouest.

Le but de cette entreprise était d'entamer sa popularité auprès des Berlinois de l'Est. Ce qui avait été auparavant présenté chez elle comme une forme d'impuissance face aux Alliés ${ }^{45}$ était désormais interprété comme une contribution active à la division de Berlin et de l'Allemagne. ${ }^{46}$ La publication non-commentée des ordres du commandant militaire du secteur soviétique de Berlin à l'adresse de Louise Schroeder participait de la même volonté de la représenter en simple exécutante des Alliés ${ }^{47}$. Tous ses actes étaient dorénavant perçus comme relevant de la trahison. ${ }^{48}$ Elle apparaissait ainsi en «mauvaise mère », se rendant coupable de négligence et d'abandon envers les Berlinois. Les journaux dénonçaient ses voyages incessants à l'Ouest, et finalement son départ pour le Bundestag ${ }^{49}$. Son séjour à 1'hôpital de Hambourg, où elle subit une opération chirurgicale, fut décrit comme la «fuite de Louise Schroeder hors de

42 «Aus der Berliner SPD». Neues Deutschland, 4 janvier 1948 ; «Frau Schroeder und die SPD». Berliner Zeitung, 6 mai 1948.

43 « Gemeinsame Front». Berliner Zeitung, 28 octobre 1947 ; « Frankfurts Oberbürgermeister beim Magistrat ». Neues Deutschland, 4 mars 1948.

44 «Der Schatten ». Neues Deutschland, 30 novembre 1947 ; « Nicht mitschuldig werden an der Spaltung. Je schneller den Zerstörern Berlins das Handwerk gelegt wird, desto besser ». Berliner Zeitung, 27 novembre 1948 ; «Taten entscheiden, Frau Louise Schroeder! ». Neues Deutschland, 4 juin 1949 ; « Wahrheit - nicht genau genommen ». Berliner Zeitung, 6 août 1948.

45 «SPD entzieht sich der Verantwortung ». Neues Deutschland, 12 juillet 1947 ; « Hoffen wir das Beste, liebe Leser! ». Berliner Zeitung, 2 août 1947.

46 «Sondersitzung im Stadthaus. Stadtparlament und die Währungsreform in Westdeutschland». Berliner Zeitung, 20 juin 1948.

47 «Auszahlung der Guthaben ab Sonntag. Einlösung ohne jede Beschränkung / Eine Anordnung General Kotikows». Berliner Zeitung, 14 août 1948 ; «General Kotikow für Ordnung in Berlin. Ein Schreiben des sowjetischen Kommandanten an die Oberbürgermeisterin ». Neues Deutschland, 25 août 1948.

${ }^{48}$ Matern, Hermann. «Was weiter - Berliner? ». Neues Deutschland, 11 juillet 1948 ; « Frau Louise Schroeder... » Neues Deutschland, 16 juillet 1948 ; «Fünf Fragen an Frau Louise Schroeder ». Neues Deutschland, 18 juillet 1948.

49 «Louise Schroeder geht». Berliner Zeitung, 5 août 1949 ; «Louise Schroeder gibt auf ». Neues Deutschland, 5 août 1949 . 
Berlin ${ }^{50}$. Le méfait le plus grave qui lui était reproché était d'avoir abusé de la confiance des Berlinoises en rencontrant Winston Churchill à Strasbourg, à l'occasion de la réunion du Conseil de l'Europe en août 1950 :

Posons la question aux nombreuses femmes dont les familles ont été bombardées : avezvous jamais pensé charger Louise Schroeder ou qui que ce soit d'autre de faire cause commune avec un homme dont les tapis de bombes en 1943, 1944, 1945 ont mis en ruine votre logement et qui menace encore de larguer des bombes atomiques, qui extermineraient toute vie à Berlin ? ${ }^{51}$

Ces attaques ont pu conduire Louise Schroeder à envisager de faire racheter la Berliner Zeitung par la municipalité, sous l'autorité de laquelle elle était officiellement placée depuis juillet $1945^{52}$. Elle dut toutefois abandonner cette idée devant l'impossibilité de la procédure - qui nécessitait l'accord de tous les sociétaires, Soviétiques inclus.

À partir du début des années 1950, Louise Schroeder disparut peu à peu des pages de la presse de la RDA. Le laconisme de sa notice nécrologique publiée dans Neues Deutschland«Louise Schroeder est morte hier à l'âge de 70 ans. Elle fut adjointe au maire de Berlin-Ouest de 1949 à $1951 »^{53}$ - laisse penser qu'on souhaitait faire oublier au lecteur l'affection que lui avaient vouée les Berlinois.

Louise Schroeder connut un destin médiatique inhabituel pour la première génération de femmes politiques allemandes. Si son action comme sa personne trouvèrent peu de résonance dans la presse, conformément au traitement médiatique réservé aux femmes de sa génération, il ne s'en est pas moins constitué, à l'Ouest, un «mythe Schroeder », parangon de vertu et de sacrifice de soi, relayé dans les décennies qui suivirent par une historiographie partiellement

\footnotetext{
50 «Louise Schroeder im Hamburger Senat?». Neues Deutschland, 10 octobre 1948.

51 «Fragen wir die vielen Frauen ausgebombter Familien: Habt ihr jemals daran gedacht, die Louise Schroeder oder sonst wen zu beauftragen, mit einem Manne gemeinsame Sache zu machen, der 1943, 1944, 1945 durch Bombenteppiche eure Wohnungen in Trümmern legen ließ und der jetzt sogar mit Atombomben droht, die alles Leben in Berlin vernichten würden?», «Zu wem fuhr Louise Schroeder? ». Neues Deutschland, 15 août 1950.

${ }^{52}$ Landesarchiv Berlin, C Rep 101-139 Oberbürgermeister. Verbindung mit Presse und Rundfunk. Magistrat von Groß-Berlin, Abteilung für Rechtswesen. Lettre à Louise Schroeder, «Erwerb der Berliner Zeitung durch GroßBerlin », 9 juin 1947.

53 «Im Alter von 70 Jahren verstarb gestern Louise Schroeder. Sie war in den Jahren 1949 bis 1951 Stellvertreter des Oberbürgermeisters in Westberlin. », «Louise Schroeder gestorben ». Neues Deutschland, 5 juin 1957.
} 
hagiographique. ${ }^{54}$ D'un apolitisme et d'un biologisme indéniables, le titre de « mère de Berlin » permettait de rendre la sociale-démocrate davantage conforme aux représentations traditionnelles qui avaient cours dans les années 1950. À l'Est, les efforts entrepris pour contenir cette image positive et marquer Schroeder du sceau de la trahison ne sont pas sans rappeler les manœuvres visant à masquer l'enthousiasme suscité en RDA par un autre social-démocrate qui avait également été maire de Berlin et n'était autre que Willy Brandt ${ }^{55}$.

Le peu de traces laissées dans la presse par Louise Schroeder, qui fut pourtant une des personnalités les plus populaires de l'après-guerre, permet de comprendre comment les femmes, qui avaient joué un rôle déterminant dans la reconstruction de l'Allemagne, ont pu être évincées si rapidement du devant de la scène politique dans les premières décennies des deux États allemands.

${ }^{54}$ Cf. par exemple Koerfer, Marthina, op. cit. ; Michalski, Bettina : Louise Schroeders Schwestern. Berliner Sozialdemokratinnen der Nachkriegszeit. Bonn: Dietz, 1996.

${ }_{55}$ Schönfelder, Jan, Erices, Rainer : Willy Brandt in Erfurt: das erste deutsch-deutsche Gipfeltreffen 1970. Berlin : Links, 2010, pp. 233-236. 